\title{
Monitoring and Treatment of Coagulation Disorders in End-Stage Liver Disease
}

\author{
Fuat H. Saner Carmen Kirchner \\ Department of General, Visceral and Transplant Surgery, University Hospital, University Duisburg-Essen, Essen, Germany
}

\section{Keywords}

End-stage liver disease, ESLD · Bleeding ·

Coagulation management

\section{Summary}

Background: Patients with end-stage liver disease (ESLD) are assumed to be at high risk of bleeding when undergoing any kind of invasive intervention (any kind of operation, including transplantation or minimally invasive interventions). Both bleeding and thrombosis are associated with a poor outcome. Methods: A selective literature research was conducted with the following key words: 'cirrhosis', 'coagulation', 'bleeding', 'INR' (international normalized ratio), 'aPTT' (activated partial thromboplastin time), and 'thrombocytopenia'. PubMed was used as the basic database. Results: Pathological values of standard laboratory tests (SLT) and thrombocytopenia have traditionally been regarded as indicators of a high risk for bleeding in all patients, and especially in those with ESLD. However, this approach has been challenged in recent years. The conventional approach in assessing a bleeding risk was based on pathological values of SLT. A 1.5 -fold increase of INR or aPTT or platelets $<50 / \mathrm{nl}$ is assumed as pathological. The traditional approach of reducing the risk of excessive bleeding during an invasive procedure was to transfuse fresh frozen plasma (FFP) or platelet concentrates in order to improve hemostasis and to avoid bleeding complications. In the recent 20 years, several studies have provided us with a basis for questioning this approach. Their results indicated that SLT were not able to predict hypocoagulation and bleeding complications. Moreover, transfusion of various blood products has been associated with an increased risk for acute lung injury, transfusion-associated circulation overload, bacterial infections, and modulation of the immune system with increased numbers of nosocomial infections. Furthermore, a high volume overload, which is required to correct a hemostasis disorder if FFP are being used in ESLD patients, may increase portal venous pressure. This might significantly increase bleeding in these ESLD patients. Although the first publication about the successful use of a viscoelastic test (VET) in liver transplantation dates back to 1985 , physicians are still very reluctant to use VETs (Thrombelastography ${ }^{\mathrm{TM}}$ and/or ROTEMTM) for the perioperative optimization of hemostasis. However, some very recent studies demonstrated that the use of VETs for assessing the risk of bleeding avoids futile transfusion with a similar safety profile. The implementation of ROTEM-based coagulation management and the use of coagulation factors (prothrombin complex, fibrinogen concentrate) have led to a highly significant reduction of FFP and red blood cell transfusions, without an increased incidence of thrombosis or bleeding. Conclusion: Patients with ESLD often show pathological values of conventional parameters used to analyze coagulation hemostasis. Without overt signs of excessive bleeding, however, they do not require coagulation treatment. The use of FFP, which is associated with fluid overload and increase in portal venous pressure, should be avoided. The preferable coagulation treatment should be based on VETguided administration of coagulation factor concentrates.

(C) 2016 S. Karger GmbH, Freiburg

\section{Hemostasis in Cirrhosis}

Hemostasis is a very complex system which is based on the interaction of platelets, endothelial cells, and plasmatic coagulation factors as well as their inhibition loops [1,2]. Hemostasis can be stratified into primary and secondary hemostasis [3]. Under physiologic conditions, endothelium and platelets are negatively charged and repulse each other. As soon as a vessel wall is damaged, subendothelium, which is positively charged, attracts the negatively

\section{KARGER}

() 2016 S. Karger GmbH, Freiburg
Prof. Dr. med. Fuat H. Saner

Klinik für Allgemein-, Viszeral- und Transplantationschirurgie Universitätsklinikum Essen

Hufelandstraße 55, 47147 Essen, Germany

fuat.saner@uni-due.de 
charged platelets. Subsequently, von Willebrand factor (vWF) is expressed on subendothelial cells and binds platelets via the GPIa/ IX receptor. In the next step, GPIIb/IIIa receptors are expressed on the surface of platelets, which serves as a binding site for fibrinogen to form the initial clot. This process is defined as primary hemostasis. During and at the end of this process, tissue factor (TF) molecules are released from endothelial cells and macrophages. The TF molecules start the secondary hemostasis with the plasmatic coagulation factor cascade [3].

Patients with liver cirrhosis suffer from general protein synthesis impairment. One of the consequences is a deficiency of vitamin $\mathrm{K}$-dependent coagulation factors II, VII, IX, and X. The proteins C and $\mathrm{S}$, which are also vitamin K-dependent, are decreased as well $[2,4]$. In contrast, coagulation factors that are not produced in the liver, such as vWF and factor VIII, show increased serum activity compared to controls $[5,6]$. One of the main reasons for elevated vWF is their increased release from endothelial cells and a lower serum activity of ADAMTS 13, a metalloprotease enzyme that breaks down the vWF. This enzyme is synthesized exclusively in the liver, and its plasma level is decreased in cirrhotic patients. This increase in vWF levels can at least in part compensate for the thrombocytopenia which frequently occurs in cirrhotic patients; thus, the primary hemostasis is preserved. Tripodi et al. [7] evaluated the endogenous thrombin potential (ETP) in cirrhotic patients and compared the data with healthy volunteers. Plasma from volunteers initially showed a higher ETP compared to cirrhotic patients. However, when thrombomodulin, a thrombin-binding molecule on the endothelial cells, was added to the plasma samples, this difference disappeared. When thrombin binds to thrombomodulin, protein $\mathrm{C}$ and $\mathrm{S}$ are activated which in turn inactivate factors V and VIII. The data of this study indicated that the activity of both pro- and anticoagulants is decreased and that this results in a new hemostasis balance. This hemostasis is not as stable as the one in healthy volunteers; however, hemostatic treatment is still not necessary.

Patients with cirrhosis have an increased risk for thrombosis. Ben-Ari et al. [8] have already observed in 1997 that patients with cholestatic liver disease showed significant hypercoagulability when compared to healthy volunteers. Their findings were supported by a study focusing on data of a registry databank from Denmark in which all patients with thrombosis and pulmonary embolism were recorded [9]. The data indicated a higher relative risk: 2.06 (95\% confidence interval (CI): 1.79-2.38) for liver cirrhosis and 2.10 (95\% CI: 1.91-2.31) for non-cirrhotic liver disease when compared to patients without pathological liver conditions.

\section{Coagulation Tests in Cirrhosis}

\section{Standard Laboratory Coagulation Assessment}

The bleeding risk is typically assessed by means of standard laboratory tests (SLT), prothrombin time (PT), and activated partial thrombin time.
PT assay was developed for the follow-up of patients therapeutically anticoagulated with oral vitamin $\mathrm{K}$ antagonists [10]. This test is performed by using a blood sample which is centrifuged, with the plasma being subsequently removed. The time between the addition of TF and calcium and the occurrence of fibrin leafs (start of coagulation) is measured. The main drawback of this coagulation assessment is that only the procoagulant but not the anticoagulant activity is being measured, as in the case of protein $\mathrm{C}$ and $\mathrm{S}$ activity [4]. Thus, the interaction between procoagulant (factors II, VII, IX, $\mathrm{X}$ ) and anticoagulant hemostatic components (protein C and S) and platelets cannot be assessed with this standard laboratory analysis. In liver cirrhosis, both pro- and anticoagulants are decreased. An increased international normalized ratio (INR) suggests a higher risk of bleeding, although the anticoagulants are also decreased, thus forming a new hemostatic balance. Many studies indicated that SLT do not reflect the actual risk of bleeding [11-14]. Ewe et al. [12] conducted a very interesting study more than 30 years ago. They evaluated the correlation between PT and spontaneous bleeding time in cirrhotic patients undergoing a laparoscopic liver biopsy. Their data clearly demonstrated that there was no correlation between actual liver bleeding time and PT. Around $40 \%$ of the patients with a PT of $100 \%$ experienced a prolonged bleeding, while patients with a PT of $15-20 \%$ had a normal liver bleeding time. Tripodi et al. [7] conducted another cornerstone study in which they demonstrated that both parts of the coagulation system, i.e. pro- and anticoagulants, showed the same level of decreased activity. These data are in line with the study of Lisman et al. [15] who measured a normal ETP during liver transplant procedure when compared to ETP in healthy controls, despite prolonged conventional coagulation tests in liver transplant patients.

A recent meta-analysis evaluated the value of SLT in terms of bleeding risk [16]. The authors analyzed more than 1,100 publications and retrieved 53 publications as well as 11 guidelines addressing this topic. They found only 3 prospective trials with altogether 108 patients, and none of these studies was a prospective randomized trial. The authors concluded that there is no scientifically based proof that SLT provide reliable data for predicting bleeding during invasive procedures.

\section{Viscoelastic Tests (Thrombelastography/ROTEM)}

Currently, two main viscoelastic tests (VETs) are being clinically used. One is thrombelastography (TEG ${ }^{\mathrm{TM}}$; Haemonetics, Niles, IL, USA), which was developed by the German physician Hartert in 1948 [17]. The other one is thrombelastometry (TEM) (ROTEM ${ }^{\mathrm{TM}}$ Delta; TEM International GmbH, Munich, Germany), being a technical modification of TEG. In the following, we will only briefly describe the two methods, as it is beyond the scope of this article to analyze them in detail.

The advantage of both methods is based on clot assessment in whole blood, thus taking into consideration the interaction of the pro- and anticoagulants, as well as the importance of platelets [4]. Both VET methods provide a graphic presentation of the clot for- 
Fig. 1. On the left side, the ROTEM device is depicted. The coagulation assessment is performed in the whole blood in which an oscillating $\left(4.75^{\circ}\right)$ pin is submerged. A mirror which reflects a beam of the light is mounted on the upper part of the pin. The reflected light is processed by a computer and a graphic according to the coagulation status is printed. When there is no coagulation (the pin can oscillate freely), there is a flat line. When clot formation occurs, the oscillation is impaired and the shape of the graphic changes, i.e. the higher the amplitude of the graph, the higher the clot strength.

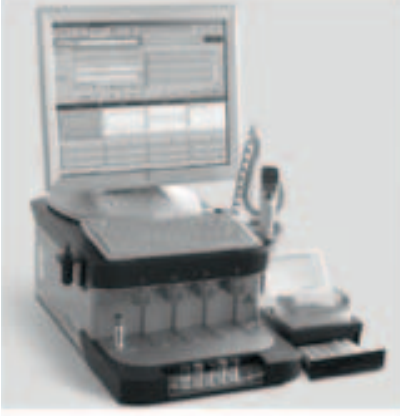

Rotem device

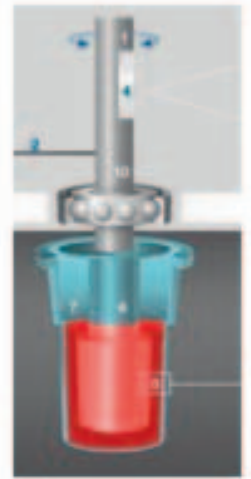

Measurement in whole blood
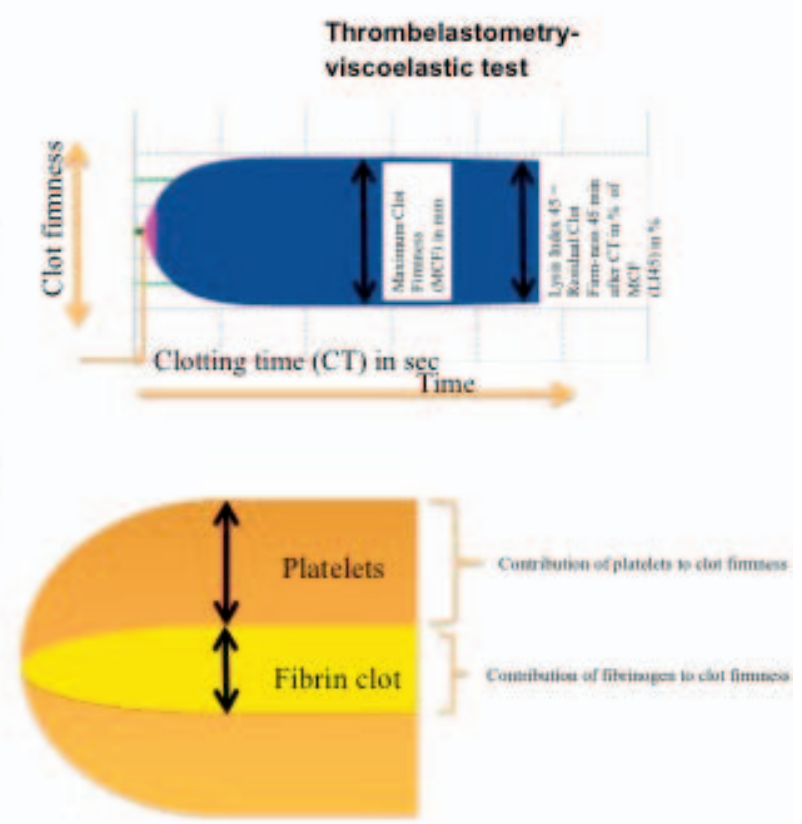

mation process, including initiation of coagulation, clot strength, and clot fibrinolysis.

\section{Thrombelastography}

For clot assessment the blood is placed in a cup and a pin is submerged in the blood. It is then activated by kaolin, and the cup starts to rotate. Initially, since there is no clot formation, the cup rotates freely. As the clot formation develops, the resistance to the cup rotation increases and the dynamics of this increase in resistance is being recorded.

Kang et al. [18] evaluated the blood coagulation system of 66 consecutive patients undergoing liver transplantation by means of TEG or SLT monitoring and assessed the first clinical use of TEG in liver transplantation. The use of TEG contributed to a $33 \%$ reduction of red blood cells (RBC), fresh frozen plasma (FFP), and platelet transfusion, whereas blood loss was comparable in all patients. Despite these very encouraging results, physicians are very reluctant to use VETs in liver transplantation.

\section{Thrombelastometry-ROTEM Delta}

TEM consists of 4 analytic channels. They are activated with 4 different substrates. Within these 4 channels, one is able to diagnose whether a patient requires platelets, fibrinogen concentrate, prothrombin complex (PCC), or antifibrinolytic treatment (e.g. tranexamic acid (TXA)) (fig. 1, 2; table 1).

Several studies indicated that TEM can be used to guide coagulation management in liver transplantation. The study conducted by Blasi et al. [19] indicated that when the amplitude (clot strength) is greater than $35 \mathrm{~mm}$ after $10 \mathrm{~min}$ (A10 (EXTEM)), the patients are unlikely to bleed to a degree that would require transfusion. Two other studies $[20,21]$ illustrated that the amplitude after 10

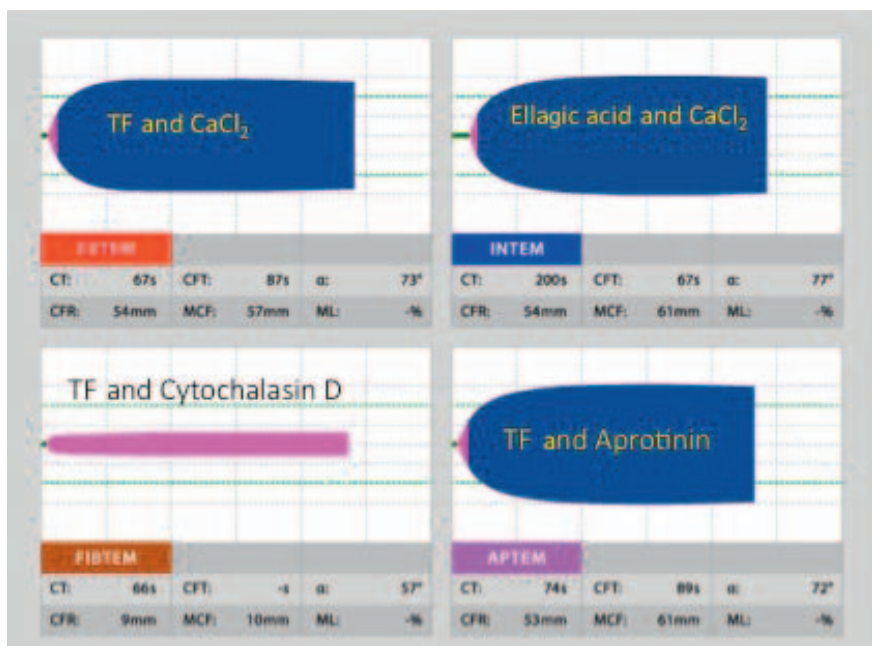

Fig. 2. Normal clot: EXTEM CT: 43-82 s; EXTEM MCF: 52-70 mm; EXTEM ML: <15\%; FIBTEM MCF: 7-24 mm; INTEM CT: 122-208 s; INTEM MCF: 51-72 $\mathrm{mm}$. There are 4 different channels, with 4 different activators. The EXTEM channel reflects the extrinsic pathway. The assay contains tissue factor and calcium chloride. The INTEM channel reflects the intrinsic pathway. Beside calcium chloride, the activator contains ellagic acid as a surface activator. The FIBTEM channel has the same activator like EXTEM but additionally includes cytochalasin D, which enables all platelets for coagulation. The assessment of the EXTEM and the FIBTEM channel enables stratification if platelets or fibrinogen concentrate is needed. If the MCF in EXTEM as well as MCF in FIBTEM is low, fibrinogen concentrate needs to be replaced. If FIBTEM is normal and MCF in EXTEM is low, platelets should be replaced in a bleeding patient. APTEM is activated with the same activator like EXTEM but contains additional aprotinin. If a clot breaks down in EXTEM and clot firmness is normal in APTEM, the patient suffers from fibrinolysis and should be treated with antifibrinolytic drugs.

min and even after 5 min shows an excellent correlation with maximum clot firmness (MCF), which enables the physician to reach an 
Table 1. Possible hemostatic disorder correlated with pathological ROTEM values

\begin{tabular}{lll}
\hline Measurements & ROTEM & Hemostatic factors \\
\hline Clot initiation & CT in s & $\begin{array}{l}\text { enzymatic coagulation factors, } \\
\text { anticoagulants, fibrin degradation } \\
\text { products, tissue factor expression }\end{array}$ \\
\hline Clot kinetics & $\begin{array}{l}\text { CFT in s; } \\
\alpha \text {-angle in degrees }\end{array}$ & $\begin{array}{l}\text { enzymatic coagulation factors, } \\
\text { anticoagulants, fibrinogen, } \\
\text { platelets }\end{array}$ \\
\hline Clot strength & A5/A10 in mm; & platelets, fibrinogen, factor XIII \\
\hline Clot stability (lysis) & $\begin{array}{l}\text { LI60 in \% of MCF; } \\
\text { ML during run } \\
\text { time in \% of MCF }\end{array}$ & $\begin{array}{l}\text { fibrinolytic enzymes, fibrinolysis } \\
\text { inhibitors, factor XIII }\end{array}$ \\
\hline
\end{tabular}

$\mathrm{CT}=$ Clotting time; $\mathrm{CFT}=$ clot formation time; $\mathrm{A} 5 / \mathrm{A} 10=$ amplitude $5 / 10 \mathrm{~min}$ after CT); MCF = maximum clot firmness; LI60 = lysis index 60 min after CT; $\mathrm{ML}=$ maximum lysis.

adequate treatment decision within 7-12 min. Another study indicated significantly lower transfusion requirements if the coagulation management was performed based on the analysis of coagulation factors and TEM. Using these tests reduced the transfusion requirement to a median of 2,0 , and 0 units for $\mathrm{RBC}, \mathrm{FFP}$, and platelets, respectively. Almost $40 \%$ of transplantations were performed without any kind of blood product, $85.3 \%$ without FFP, and $71.4 \%$ without platelets.

Depending on the new coagulation model [3], thrombin generation is a physiological process which consists of three stages: initiation, propagation, and amplification. Following a burst of thrombin activation, fibrin is being produced from fibrinogen to build a clot which will be strengthened by factor XIII.

SLT assess only the initial $10 \%$ of thrombin generation whereas VETs will assess the whole clot formation, including the first $10 \%$, the clot strength, and fibrinolysis (fig. 3).

However, the whole coagulation process (initiation, propagation, and amplification) is followed by VETs.

\section{Bleeding Management in Patients with End-Stage Liver Disease Undergoing Invasive Procedures}

During the process of bleeding management it is important not only to control the bleeding but also to prevent the development of thrombosis.

\section{Coagulation Management in Patients with Cirrhosis Scheduled for Invasive Procedure}

The traditional approach for the management of the hemostatic system has consisted of proactive transfusion based on SLT values

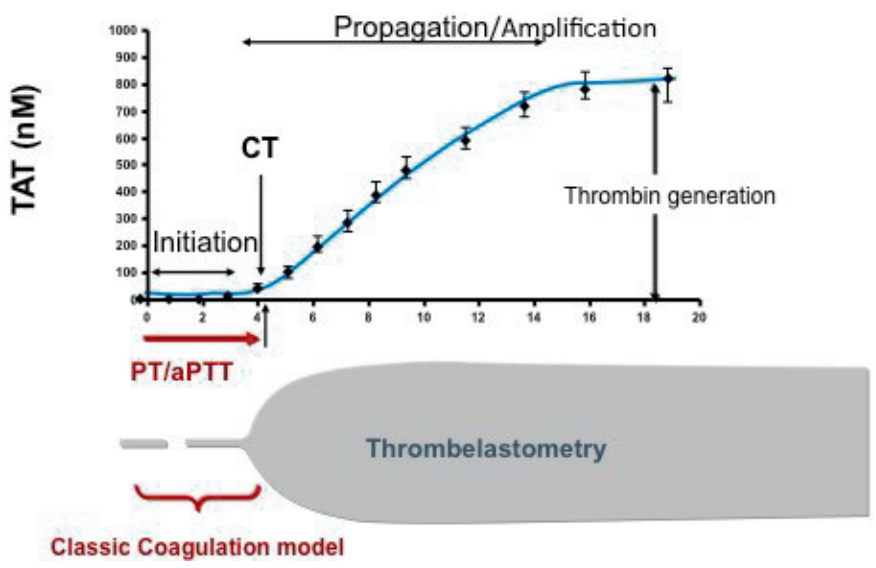

Fig. 3. The new hypothesis of the coagulation cascade stratifies the coagulation process into initiation, propagation, and amplification. Fibrin formation will occur at the end of the process. The initiation process reflects only the very early beginning of coagulation and correlates with the first $5-10 \%$ of thrombin generation. The SLT assess only these $5-10 \%$; further clot development and clot strength will not be assessed. In contrast to the SLT, the VETs assess the initiation of the coagulation, the acceleration, the clot strength, and finally, if it occurs, also the fibrinolysis.

in order to try to prevent a bleeding complication [4]. However, based on the aforementioned studies which used VETs as a guiding parameter, the newly developed concept of balanced coagulation suggests that proactive coagulation treatment is not necessary unless excessive bleeding develops [1].

\section{Bleeding Management in End-Stage Liver Disease}

According to the concept of a new rebalanced hemostasis, it includes a more conservative attitude towards transfusion of RBC, FFP, or platelets. Unnecessary transfusion of these blood components should be avoided since we do not have any scientific proof of a benefit associated with these SLT-guided transfusions [22]. Two major randomized controlled trial (RCT) studies in patients with end-stage liver disease (ESLD) explicitly demonstrate that the use of VETs can significantly reduce the amount of transfusion without an increase of bleeding events. Wang et al. [23] conducted an RCT study in which 28 patients were assigned to VET- versus SLTguided hemostatic management. In patients monitored via TEG, significantly less FFP was transfused (mean: 12.8 vs. 21.5 units). Importantly, the 3-year survival in both groups did not differ.

De Pietri et al. [24] conducted an RCT in 60 patients with ESLD who were scheduled for various invasive surgical interventions (laparoscopy, liver biopsy, liver resection). The patients were randomly assigned to VET and SLT groups in a ratio of 1:1. Coagulopathy was defined as INR $\geq 1.8$ and platelet count $\leq 50 / \mathrm{nl}$. These values acted as a trigger for FFP or platelet transfusion. TEG was used as a VET method. If the reaction time (i.e. time required from clot initiation to form $2 \mathrm{~mm}$ deviation, expressed in min, equals clotting time (CT) in ROTEM) was pathological, FFP were transfused to correct the hemostasis; if the maximum amplitude was 
$\leq 40 \mathrm{~mm}$, then platelets were transfused. In the control group, $100 \%$ of the patients received at least one of the blood components (RBC, FFP, and/or platelet), as compared to only $16 \%$ in the TEG group. Clinically significant bleeding episodes were more frequently observed in the SLT group; however, their number did not reach statistical significance.

Leon-Justel et al. [25] conducted a prospective trial in 200 liver transplantation patients. The first 100 patients were treated with an SLT-guided hemostatic management protocol while the other 100 patients received ROTEM-guided hemostatic management. The study results indicated a significant reduction of transfused RBC, FFP, and platelets in the ROTEM group. Moreover, the number of blood product-free transplantations increased from 5 to $24 \%$ ( $\mathrm{p}<$ 0.001 ), and secondary endpoints like reoperation for bleeding, acute kidney failure, or hemodynamic instability were significantly lower in the ROTEM group.

In a prospective study, Bedreli et al. [26] showed that compared with SLT-guided hemostasis treatment, ROTEM-guided coagulation management for minimally invasive procedures can reduce the use of coagulation factors without increasing bleeding complications, providing further support for VET-guided hemostatic management.

\section{Treatment of Thrombin Generation Impairment}

Thrombin generation is the basic step in clot formation. According to the classic model of the physiology of coagulation, the coagulation cascade is divided into extrinsic and extrinsic pathways. Both pathways lead to an activation of coagulation factor X. Following the activation of the factor $\mathrm{X}$, a common pathway of activation of coagulation factors is initiated, leading to the conversion of prothrombin into thrombin. Thrombin activates the fibrinopeptids A and B which break down fibrinogen into fibrin which in turn forms a fibrin net. This fibrin net is then stabilized by factor XIII. The new model of coagulation system functioning presumes that the coagulation system consists of three phases: initiation, propagation, and amplification [3]. At the end of the amplification phase, a thrombin burst occurs, which induces formation of the fibrin net and cross-linking with factor XIII to enhance the clot strength. The activation of coagulation depends on the factors II, $\mathrm{V}$, VII, IX, and X. Deficiency of these factors is detected as a prolonged CT of the EXTEM channel (fig. 1, 2; table 1) [4]. In bleeding patients, a cut-off value of $<75 \mathrm{~s}$ seems to be an adequate value to prevent coagulopathic bleeding [27].

Two alternatives for the treatment of an impaired thrombin generation are available, namely the administration of FFP or of PCC.

There are two special requirements for a drug, i.e. efficacy and safety. Abdel-Wahab et al. [28] evaluated the efficacy of FFP in critically ill patients with moderate coagulopathy $(1.1 \leq \mathrm{INR} \leq 1.85)$. The authors calculated an INR decrease which correlated with the number of transfused FFP. Transfusion of FFP resulted in normalization of PT-INR values in only $0.8 \%$ of the patients. Even transfusion of 4 units of FFP and more did not improve hemostasis.

Sarani et al. [29] evaluated the safety of FFP transfusion in critically ill patients. Nosocomial infections occurred three times more often in patients who received FFP transfusions. In this multivariate analysis, the infection rate per transfused FFP unit was 1.039.

Stanworth et al. [30] conducted a multicenter coagulopathy study in which they analyzed the utilization of FFP in intensive care unit patients. There was a wide range of futile FFP transfusion. Based on their results, the authors concluded that in a high number of patients who received FFP a beneficial effect could not be seen.

In this context, a study is of interest in which 4-factor PCC (4F-PCC) was administered to successfully control acute bleeding in patients following/during surgery [31]. The authors administered a dose of $25 \mathrm{IU} / \mathrm{kg}$ body weight (BW) when CT EXTEM was greater than $75 \mathrm{~s}$ and of $40 \mathrm{IU} / \mathrm{kg}$ BW when CT EXTEM was greater than $100 \mathrm{~s}$. No major side effects or complications were reported. The used 4-F-PCC preparations (Beriplex ${ }^{\mathrm{TM}}$; CSL Behring, Marburg, Germany, or Octaplex ${ }^{\mathrm{TM}}$; Octapharma, Lachen, Switzerland) contain vitamin K-dependent procoagulants (factor II, VII, IX, and X) as well as vitamin K-dependent anticoagulants (protein $\mathrm{C}$ and $\mathrm{S}$ ) and are considered to be safe regarding the risk of thrombosis [32]. The first publication reporting the use of 4-F-PCC in ESLD dates back to 1994 [33]. Initially, 4-F-PCC was applied together with antithrombin 3 (AT III) to minimize the risk of thrombosis. However, if PCC is only being used in bleeding patients and the usage is guided by TEG and with high concentration of protein $\mathrm{C}$ and $\mathrm{S}$ in the vials, the use of AT III might not be necessary. In our opinion, PCC administration should be guided by TEG to avoid overtreatment and thrombosis. A recent casecontrol study in 256 liver transplant patients indicated that the use of coagulation factors, including 4-F-PCC, did not increase the rate of thrombotic events compared with patients receiving no coagulation factors [31].

\section{Management of Clot Firmness Impairment}

Clot firmness depends on the interaction of fibrinogen and platelets. Fibrinogen is the most important coagulation factor. More than $90 \%$ of the coagulation factors are mapped from fibrinogen, which is the only coagulation factor to be kept within a normal range to build a stable clot. The interaction between platelets and fibrinogen should be thought of as a brick wall, whereas the bricks are supposed to be the platelets and the fibrinogen is the cement which keeps the bricks together [34].

The most valuable diagnostic method for clot firmness assessment is ROTEM. The MCF in the EXTEM channel provides the best information about the interaction of platelets and fibrinogen. The FIBTEM channel offers information on the sole fibrinogen contribution to the whole clot formation. The values/graphic presentation/major parameters (CT, MCF) obtained from these two ROTEM channels give us information whether the patient requires fibrinogen and/or platelets [35]. In case of excessive bleeding and low MCF in EXTEM and FIBTEM, fibrinogen should be applied; if MCF in FIBTEM is within the normal range, then platelets should be replaced.

A recent study from our group indicated that an MCF $\geq 40 \mathrm{~mm}$ in EXTEM and an MCF $\geq 9 \mathrm{~mm}$ in FIBTEM are safe margins to avoid bleeding in liver transplantation [31]. 
Fig. 4. a Fibrinolysis after reperfusion. The EXTEM shows a very slight clot forming with immediate breakdown. The FIBTEM channel indicates that no fibrinogen is available (flat line). The APTEM channel indicates that adding aprotinin is able to maintain a weak clot building only from the platelets. After replacement of TXA and fibrinogen bleeding could be stopped. b Example of platelet requirement. MCF in EXTEM is decreased, while it is normal in FIBTEM. c In all 3 channels, i.e. EXTEM, INTEM, and FIBTEM, the clot is broken down. After 30 min a flat line is detected in all channels, which indicates no clotting. ML shows $100 \%$ which means that the whole clot undergoes lysis. The APTEM channel contains aprotinin, an antifibrinolytic drug, and indicates that lysis is stopped by antifibrinolytic treatment in vivo. In a bleeding patient, the indication for antifibrinolytic therapy, e.g. with TXA, is given.

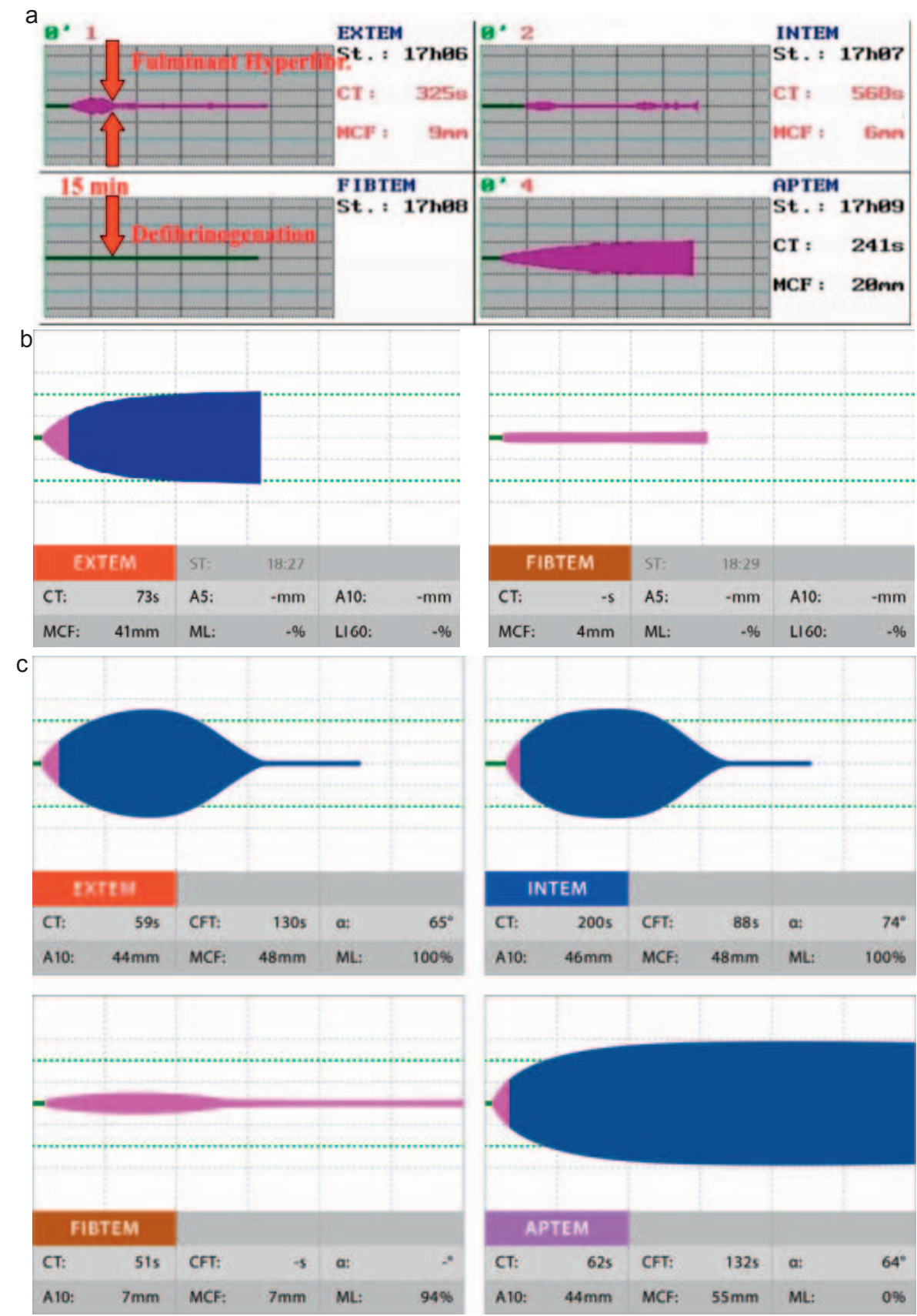

There are some recent studies which showed that already after 10 or even $5 \mathrm{~min}$, an MCF value can be used to decide about administering fibrinogen or platelets. Görlinger et al. [21] showed that early values of clot firmness in EXTEM and FIBTEM (after 5, 10 , and $15 \mathrm{~min}$ ) allow for a fast and reliable prediction of ROTEM $\mathrm{MCF}$ in non-cardiac surgery patients. Song et al. [20] reported that in liver transplant patients, ROTEM parameters are sufficient to assess the requirement of platelet transfusion and/or hypofibrinogenemia as soon as 5 min after starting the test. Blasi et al. [19] showed in their study that the clot formation amplitude after 10 min in EXTEM (A10 (EXTEM)) is a specific and reliable parameter for coagulation management. Patients with A10 (EXTEM) $>35$ $\mathrm{mm}$ - with A10 indicating the clot amplitude $10 \mathrm{~min}$ after the end of CT - are unlikely to suffer from excessive bleeding caused by a disruption of hemostasis.

\section{Detection and Management of Fibrinolysis}

The gold standard for the assessment of fibrinolysis are VETs [36]. Figure 4 presents fibrinolysis as detected by the ROTEM method. In the EXTEM, INTEM, and FIBTEM, graphic presentation of clot stability depicts fibrinolysis (after reaching the maximum amplitude the clot breaks down and, consequently, the am- 
plitude on the graph is smaller). The ratio (MCF-Amplitude ${ }_{\text {At time- }}$ point of maximum lysis /MCF expressed as $\%$ ) is called maximum lysis (ML). Excessive fibrinolysis is assumed when ML is $\geq 15 \%$.

Hyperfibrinolysis in liver transplantation has been reported to occur very frequently (range 5-84\%), mainly during reperfusion of the transplanted liver $[37,38]$. However, most of the fibrinolysis is self-limiting and should only be treated when it occurs concomitantly with excessive bleeding [38]. The most commonly used antifibrinolytic drug is TXA. When fibrinolysis in combination with excessive bleeding occurs, TXA (30 mg/kg) should be administered, and ROTEM control should be performed $30 \mathrm{~min}$ after the administration of TXA.

\section{Conclusion}

Hemostasis in ESLD is a very complex system in a new rebalanced homeostasis. If patients do not bleed acutely, prophylactic coagulation management should be avoided, especially since available data indicate a higher risk of thrombosis in cirrhotic patients as compared to the normal population.

Coagulation management should be based on VET analysis because this kind of coagulation analysis reflects coagulation dynamics better, enables a faster reaction to an imbalance in the coagulation system, and is the gold standard for detecting fibrinolysis.

Based on the current data, hepatic coagulopathy should be managed with coagulation factors rather than with FFP, as they are more effective and have fewer side effects in terms of potential infection and/or patient volume overload.

\section{Disclosure Statement}

FH Saner: CSL Behring: Honoraria from speakers bureau; TEM International: research grant.

\section{References}

1 Schaden E, Saner FH, Görlinger K: Coagulation pattern in critical liver dysfunction. Curr Opin Crit Care 2013;19:142-148.

2 Tripodi A, Mannucci PM: The coagulopathy of chronic liver disease. N Engl J Med 2011;365:147-156.

3 Hoffman M, Monroe DM 3rd: A cell-based model of hemostasis. Thromb Haemost 2001;85:958-965.

4 Saner FH, Gieseler RK, Akiz H, Canbay A, Görlinger $\mathrm{K}$ : Delicate balance of bleeding and thrombosis in endstage liver disease and liver transplantation. Digestion 2013;88:135-144.

5 Pereboom IT, Adelmeijer J, van Leeuwen Y, Hendriks HG, Porte RJ, Lisman T: No evidence for systemic platelet activation during or after orthotopic liver transplantation. Liver Transpl 2009;15:956-962.

6 Lisman T, Bongers TN, Adelmeijer J, Janssen HL, de Maat MP, de Groot PG, Leebeek FW: Elevated levels of von Willebrand Factor in cirrhosis support platelet adhesion despite reduced functional capacity. Hepatology 2006;44:53-61.

7 Tripodi A, Salerno F, Chantarangkul V, Clerici M, Cazzaniga M, Primignani M, Mannuccio Mannucci $\mathrm{P}$ Evidence of normal thrombin generation in cirrhosis despite abnormal conventional coagulation tests. Hepatology 2005;41:553-558.

8 Ben-Ari Z, Panagou M, Patch D, Bates S, Osman E, Pasi J, Burroughs A: Hypercoagulability in patients with primary biliary cirrhosis and primary sclerosing cholangitis evaluated by thrombelastography. J Hepatol 1997; 26:554-559.

9 Søgaard KK, Horváth-Puhó E, Grønbaek H, Jepsen P, Vilstrup H, Sørensen HT: Risk of venous thromboembolism in patients with liver disease: a nationwide population-based case-control study. Am J Gastroenterol 2009;104:96-101.

10 Owren PA, Aas K: The control of dicumarol therapy and the quantitative determination of prothrombin and proconvertin. Scand J Clin Lab Invest 1951;3:201208
Segal JB, Dzik WH; Transfusion Medicine/Hemostasis Clinical Trials Network: Paucity of studies to support that abnormal coagulation test results predict bleeding in the setting of invasive procedures: an evidencebased review. Transfusion 2005;45:1413-1425.

12 Ewe K: Bleeding after liver biopsy does not correlate with indices of peripheral coagulation. Dig Dis Sci 1981;26:388-393.

13 Terjung B, Lemnitzer I, Dumoulin FL, Effenberger W, Brackmann HH, Sauerbruch T, Spengler U: Bleeding complications after percutaneous liver biopsy. An analysis of risk factors. Digestion 2003;67:138-145.

14 Boks AL, Brommer EJ, Schalm SW, Van Vliet HH: Hemostasis and fibrinolysis in severe liver failure and their relation to hemorrhage. Hepatology 1986;6:79-86.

15 Lisman T, Bakhtiari K, Pereboom IT, Hendriks HG, Meijers JC, Porte RJ: Normal to increased thrombin generation in patients undergoing liver transplantation despite prolonged conventional coagulation tests. J Hepatol 2010;52:355-361.

16 Haas T, Fries D, Tanaka KA, Asmis L, Curry NS, Schochl H: Usefulness of standard plasma coagulation tests in the management of perioperative coagulopathic bleeding: is there any evidence? Br J Anaesth 2015;114:217-224.

17 Hartert H: Blutgerinnungsstudien mit der Thrombelastographie, einem neuen Untersuchungsverfahren. Klin Wochenschr 1948;26:577-583.

18 Kang YG, Martin DJ, Marquez J, Lewis JH, Bontempo FA, Shaw BW Jr, Starzl TE, Winter PM: Intraoperative changes in blood coagulation and thrombelastographic monitoring in liver transplantation. Anesth Analg 1985;64:888-896

19 Blasi A, Beltran J, Pereira A, Martinez-Palli G, Torrents A, Balust J, Zavala E, Taura P, Garcia-Valdecasas JC: An assessment of thromboelastometry to monitor blood coagulation and guide transfusion support in liver transplantation. Transfusion 2012;52:1989-1998.
20 Song JG, Jeong SM, Jun IG, Lee HM, Hwang GS: Fiveminute parameter of thromboelastometry is sufficient to detect thrombocytopenia and hypofibrinogenaemia in patients undergoing liver transplantation. $\mathrm{Br} \mathrm{J}$ Anaesth 2014;112:290-297.

21 Görlinger K, Dirkmann D, Solomon C, Hanke AA: Fast interpretation of thromboelastometry in non-cardiac surgery: reliability in patients with hypo-, normo-, and hypercoagulability. Br J Anaesth 2013;110:222-230.

22 Stanworth SJ, Grant-Casey J, Lowe D, Laffan M, New $\mathrm{H}$, Murphy MF, Allard S: The use of fresh-frozen plasma in England: high levels of inappropriate use in adults and children. Transfusion 2011:51:62-70.

23 Wang SC, Shieh JF, Chang KY, Chu YC, Liu CS, Loong CC, Chan KH, Mandell S, Tsou MY: Thromboelastography-guided transfusion decreases intraoperative blood transfusion during orthotopic liver transplantation: randomized clinical trial. Transplant Proc 2010; 42:2590-2593

24 De Pietri L, Bianchini M, Montalti R, De Maria N, Di Maira T, Begliomini B, Gerunda GE, di Benedetto F, Garcia-Tsao G, Villa E: Thrombelastography-guided blood product use before invasive procedures in cirrhosis with severe coagulopathy. A randomized controlled trial. Hepatology 2016;63:566-573.

25 Leon-Justel A, Noval-Padillo JA, Alvarez-Rios AI, Mellado P, Gomez-Bravo MA, Alamo JM, Porras M, Barrero L, Hinojosa R, Carmona M, Vilches-Arenas A, Guerrero JM: Point-of-care haemostasis monitoring during liver transplantation reduces transfusion requirements and improves patient outcome. Clin Chim Acta 2015;446:277-283.

26 Bedreli S, Sowa JP, Gerken G, Saner FH, Canbay A: Management of acute-on-chronic liver failure: rotational thromboelastometry may reduce substitution of coagulation factors in liver cirrhosis. Gut 2016;65: 357-358. 
27 Fayed N, Mourad W, Yassen K, Görlinger K: Preoperative thromboelastometry as a predictor of transfusion requirements during adult living donor liver transplantation. Transfus Med Hemother 2015;42:99-108.

28 Abdel-Wahab OI, Healy B, Dzik WH: Effect of freshfrozen plasma transfusion on prothrombin time and bleeding in patients with mild coagulation abnormalities. Transfusion 2006;46:1279-1285.

29 Sarani B, Dunkman WJ, Dean L, Sonnad S, Rohrbach JI, Gracias VH: Transfusion of fresh frozen plasma in critically ill surgical patients is associated with an in creased risk of infection. Crit Care Med 2008;36:11141118.

30 Stanworth SJ, Walsh TS, Prescott RJ, Lee RJ, Watson DM, Wyncoll D; Intensive Care Study of Coagulopathy (ISOC) investigators: A national study of plasma use in critical care: clinical indications, dose and effect on prothrombin time. Crit Care 2011;15:R108.
31 Kirchner C, Dirkmann D, Treckmann JW, Paul A, Hartmann M, Saner FH, Görlinger K: Coagulation management with factor concentrates in liver transplantation: a single-center experience. Transfusion 2014; 54:2760-2768.

32 Hanke AA, Joch C, Görlinger K: Long-term safety and efficacy of a pasteurized nanofiltrated prothrombin complex concentrate (Beriplex $\mathrm{P} / \mathrm{N}$ ): a pharmacovigilance study. Br J Anaesth 2013;110:764-772.

33 Scherer R, Gille A, Erhard J, Paar D, Kox WJ: The effect of substitution with AT III- and PPSB-concentrates in patients with terminal liver insufficiency (Article in German). Anaesthesist 1994;43:178-182.

34 Lang T, von Depka M: Possibilities and limitations of thrombelastometry/-graphy (Article in German). Hamostaseologie 2006;26(suppl 1):S20-29.
35 Bienholz A, Canbay A, Saner FH: Coagulation management in patients with liver disease (Article in German). Med Klin Intensivmed Notfmed 2016;111:224234.

36 Larsen OH, Fenger-Eriksen C, Christiansen K, Ingerslev J, Sorensen B: Diagnostic performance and therapeutic consequence of thromboelastometry activated by kaolin versus a panel of specific reagents. Anesthesiology 2011;115:294-302.

37 Poon KS, Chen CC, Thorat A, Chiang YY, Jeng LB, Yang HR, Chen TH, Yeh CC, Chen KB: Fibrinolysis after reperfusion of liver graft. Acta Anaesthesiol Taiwan 2015;53:41-43.

38 Görlinger K: Coagulation management during liver transplantation (Article in German). Hamostaseologie 2006;26(suppl 1):S64-76. 